\title{
GENIUS - A New Facility of Non-Accelerator Particle Physics
}

\author{
H.V. Klapdor-Kleingrothaus ${ }^{\text {a }}$ \\ ${ }^{a}$ Max-Planck-Institut für Kernphysik, P.O.Box 1039 80, D-69029 Heidelberg, Germany \\ Spokesman of HEIDELBERG-MOSCOW and GENIUS Collaborations \\ e-mail:klapdor@gustav.mpi-hd.mpg.de, home page: http://mpi-hd.mpg.de.non-acc/
}

The GENIUS (emanium in Liquid Nitrogen Underground Setup) project has been proposed in 1997 [1] as first third generation double beta decay project, with a sensitivity aiming down to a level of an effective neutrino mass of $<m>\sim 0.01-0.001 \mathrm{eV}$. Such sensitivity has been shown to be indispensable to solve the question of the structure of the neutrino mass matrix which cannot be solved by neutrino oscillation experiments alone [2]. It will allow broad access also to many other topics of physics beyond the Standard Model of particle physics at the multi-TeV scale. For search of cold dark matter GENIUS will cover almost the full range of the parameter space of predictions of SUSY for neutralinos as dark matter [3, A]. Finally, GENIUS has the potential to be the first real-time detector for low-energy ( $\mathrm{pp}$ and ${ }^{7} \mathrm{Be}$ ) solar neutrinos [6,5]. A GENIUS-Test Facility has just been funded and will come into operation by end of 2001 .

\section{Introduction}

Underground physics can complement in many ways the search for New Physics at future colliders such as LHC and NLC and can serve as important bridge between the physics that will be gleaned from future high energy accelerators on the one hand, and satellite experiments such as MAP and PLANCK on the other [7]. The first indication for beyond SM physics indeed has come from underground experiments (neutrino oscillations from SK), and this type of physics will play an even large role in the future.

Concerning neutrino physics, without double beta decay there will be no solution of the nature of the neutrino (Dirac or Majorana particle) and of the structure of the neutrino mass matrix. Only investigation of $\nu$ oscillations and double beta decay together can lead to an absolute mass scale.

Concerning the search for cold dark matter, even a discovery of SUSY by LHC will not have proven that neutralinos form indeed the cold dark matter in the Universe. Direct detection of the latter by underground detectors remains indispensable. Concerning solar neutrino physics, present information on possible $\nu$ oscillations re- lies on $0.2 \%$ of the solar neutrino flux. The total pp neutrino flux has not been measured and also no real-time information is available for the latter.

The GENIUS project proposed in 1997 (see 11, 7) as the first third generation $\beta \beta$ detector, could attack all of these problems with an unpredented sensitivity.

\section{GENIUS, Double Beta Decay and the Light Majorana Neutrino Mass}

Present double beta experiments are not able to reach a limit for the (effective) neutrino mass below $\sim 0.1 \mathrm{eV}$. The most sensitive experiment is since eight years the HEIDELBERGMOSCOW experiment using the world's largest source strength of $11 \mathrm{~kg}$ of $86 \%$ enriched ${ }^{76} \mathrm{Ge}$ in form of 5 high-purity Ge detectors, run in the Gran Sasso Underground laboratory. The limits reached after $37.24 \mathrm{~kg}$ y of measurement

$T_{1 / 2}^{0 \nu}>3.5(2.1) \cdot 10^{25} y$ and $<m_{\nu}><$ $0.26(0.34) \mathrm{eV}, 68 \%$ and $90 \%$ c.l., respectively.

The status and potential of other experiments are shown in Fig. 1.

With the era of the HEIDELBERG-MOSCOW experiment which will remain the most sensitive 


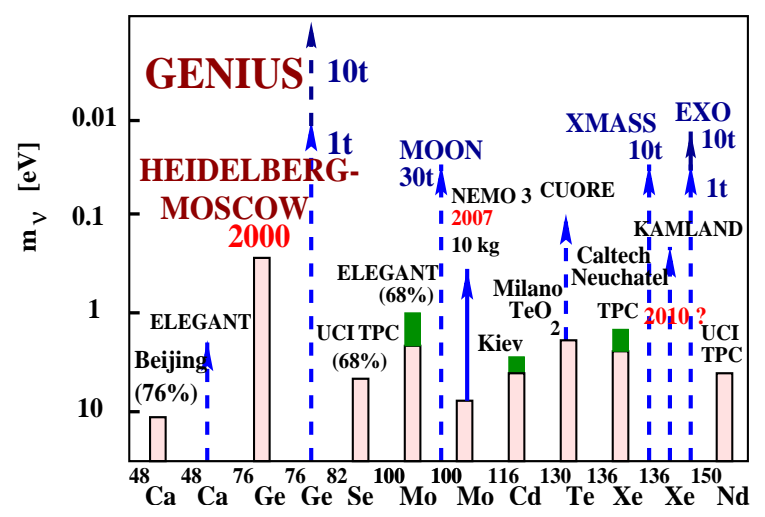

Figure 1. Present situation, 2000, and expectation for the future, of the most promising $\beta \beta$ experiments. Light parts of the bars: present status; dark parts: expectation for running experiments; solid and dashed lines: experiments under construction or proposed experiments. For references see [12].

experiment for the next years, the time of the small smart experiments is over.

The requirements in sensitivity for future experiments to play a decisive role in the solution of the structure of the neutrino mass matrix are shown in Fig.2. Shown are the expectations for the effective neutrino mass (the observable in $\beta \beta$ decay) from the present experimental status of all existing neutrino oscillation experiments in the different presently experimentally favored neutrino mass models [2, 8].

It can be seen that a sensitivity down to $<m_{\nu}>\approx 0.001 \mathrm{eV}$ as it may be reached only by the GENIUS project will be able to test all neutrino scenarios allowed by the oscillation experiments, except for one, the not favoured hierarchical LOW solution. For details see [2, 8, 9].

To reach this level of sensitivity $\beta \beta$ experiments have to become large. A source strength of up to 10 tons of enriched material touches the world production limits. At the same time the background has to be reduced by a factor of 1000 and more compared to that of the HEIDELBERG-

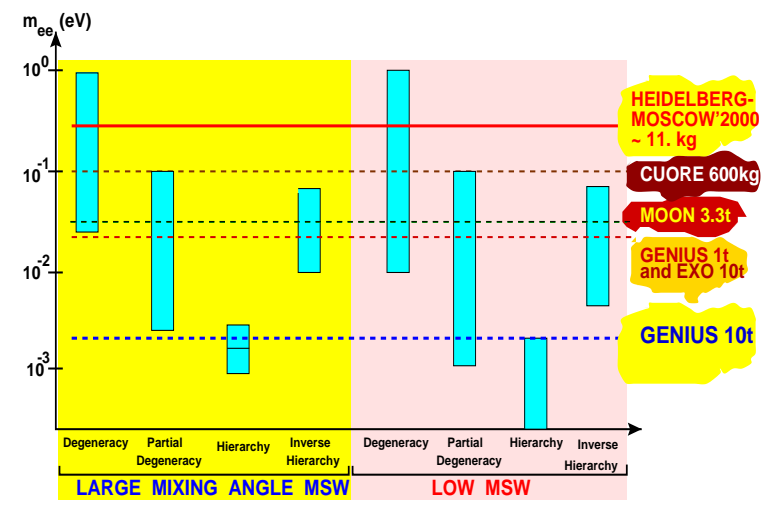

Figure 2. Values expected from $\nu$ oscillation experiments for $m_{e e} \equiv\left(<m_{\nu}>\right)$ in different schemes. The expectations are compared with the present neutrino mass limits $o b$ tained from the HEIDELBERG-MOSCOW experiment as well as the expected sensitivities for the CUORE, MOON, EXO proposals and the 1 ton and 10 ton proposal of GENIUS [8]. For references and more details about the different experiments see [7,12].

MOSCOW experiment.

Table 1 lists some key numbers for GENIUS, and of the main other proposals made after the GENIUS proposal. Their potential is shown also in Fig.2. It is seen that not all of these proposals fully cover the region to be probed. Among them is also the recently presented MAJORANA project.

In the GENIUS project a reduction by a factor of more than 1000 down to a background level of 0.1 events/tonne $\mathrm{y} \mathrm{keV}$ in the range of $0 \nu \beta \beta$ is reached by removing all material close to the detectors, and by using naked Germanium detectors in a large tank of liquid nitrogen. It has been shown that the detectors show excellent performance under such conditions [5].

For technical questions and extensive Monte Carlo simulations of the GENIUS project for its application in double beta decay we refer to [5]. 


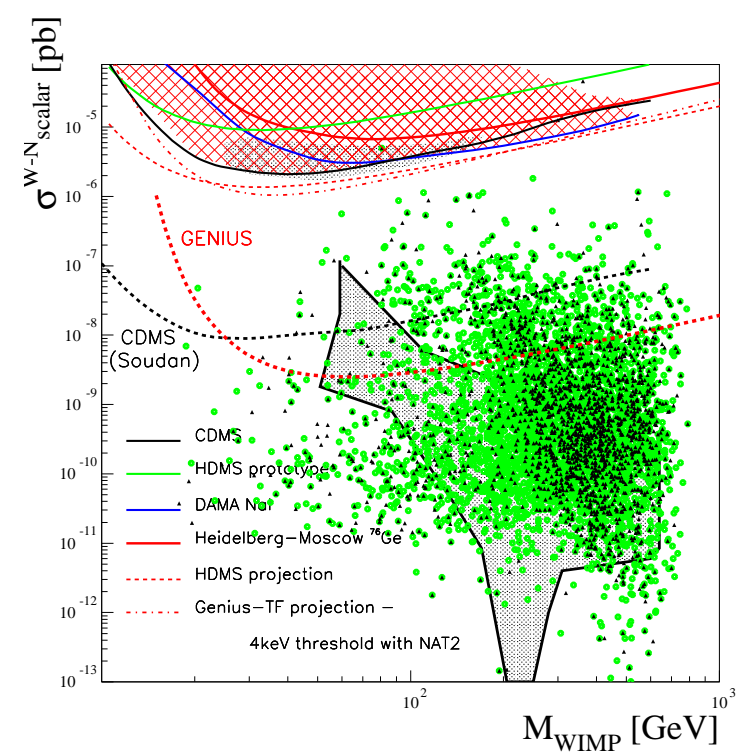

Figure 3. WIMP-nucleon cross section limits in $\mathrm{pb}$ for scalar interactions as function of the WIMP mass in GeV. Shown are contour lines of present experimental limits (solid lines) and of projected experiments (dashed lines). Also shown is the region of evidence published by DAMA. The theoretical expectations are shown by a scatter plot (from [4]) and by the grey region (from 11).

\section{GENIUS and Other Beyond Standard Model Physics}

GENIUS will allow besides the major step in neutrino physics described in section 2 the access to a broad range of other beyond SM physics topics in the multi- $\mathrm{TeV}$ range. Already now $\beta \beta$ decay probes the TeV scale on which new physics should manifest itself (see, e.g. [1, 10]). Basing to a large extent on the theoretical work of the Heidelberg group in the last four years, the HEIDELBERGMOSCOW experiment yields results for SUSY models (R-parity breaking, sneutrino mass), leptoquarks (leptoquarks-Higgs coupling), compositeness, right-handed $\mathrm{W}$ mass, nonconservation of Lorentz invariance and equivalence principle, mass of a heavy left or righthanded neutrino, competitive to corresponding results from highenergy accelerators like TEVATRON and HERA.
The potential of GENIUS extends into the multi$\mathrm{TeV}$ region for these fields and its sensitivity would correspond to that of LHC or NLC and beyond (for details see [0,10]).

\section{GENIUS and Cold Dark Matter Search}

Already now the HEIDELBERG-MOSCOW experiment is the most sensitive Dark Matter experiment worldwide concerning the raw data. GENIUS would already in a first step, with 100 $\mathrm{kg}$ of natural Ge detectors, cover a significant part of the MSSM parameter space for prediction of neutralinos as cold dark matter (Fig. 3). For this purpose the background in the energy range $<$ $100 \mathrm{keV}$ has to be reduced to $10^{-2}$ events $/ \mathrm{kg}$ y $\mathrm{keV}$, which is possible if the detectors are produced and handled on Earth surface under heavy shielding, to reduce the cosmogenic background produced by spallation through cosmic radiation (critical products are tritium, ${ }^{68} \mathrm{Ge},{ }^{63} \mathrm{Ni}, \ldots$ ) to a minimum. For details we refer to [5]. Fig. 3 shows together with the expected sensitivity of GENIUS predictions for neutralinos as dark matter by two models, one basing on supergravity [11], another starting from more relaxed unification conditions 4].

The sensitivity of GENIUS for Dark Matter corresponds to that obtainable with a 1 $\mathrm{km}^{3}$ AMANDA detector for indirect detection (neutrinos from neutralino annihilation at the Sun). Interestingly both experiments would probe different neutralino compositions: GENIUS mainly gaugino-dominated neutralinos, AMANDA mainly neutralinos with comparable gaugino and Higgsino components. It should be stressed that, together with DAMA, GENIUS will be the only future Dark Matter experiment, which would be able to positively identify a dark matter signal by the seasonal modulation signature. This cannot be achieved, for example, by the CDMS experiment.

\section{GENIUS and Low-Energy Solar Neutri- nos}

Gallex and Sage measure pp $+{ }^{7} B e+{ }^{8} B$ neutrinos $(60+30+10 \%)$ down to $0.24 \mathrm{MeV}$, the 


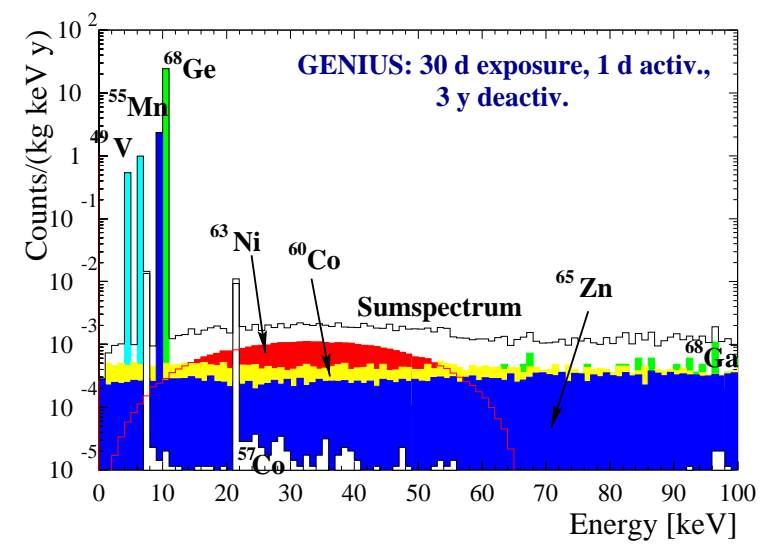

Figure 4. Simulated cosmogenic background during detector production. Assumptions: 30 days exposure of material before processing, $1 \mathrm{~d}$ activation after zone refining, 3 y deactivation underground (see [12]).

Chlorine experiment measured ${ }^{7} B e+{ }^{8} B$ neutrinos $\left(80 \%^{8} B\right)$ above $E_{\nu}=0.817 \mathrm{MeV}$, all without spectral, time and detection information. No experiment has separately measured the pp and ${ }^{7} \mathrm{Be}$ neutrinos and no experiment has measured the full pp $\nu$ flux. BOREXINO plans to measure ${ }^{7} \mathrm{Be}$ neutrinos, the access to pp neutrinos being limited by ${ }^{14} C$ contamination (the usual problem of organic scintillators). GENIUS could be the first detector measuring the full pp ( and ${ }^{7} \mathrm{Be}$ ) neutrino flux in real time.

Extending the radius of GENIUS to $13 \mathrm{~m}$ and improving some of the shielding parameters as described in [5.6] the background can be reduced to a level of $10^{-3}$ events/ $\mathrm{kg} \mathrm{y} \mathrm{keV} \mathrm{(Fig.} \mathrm{4)}$ (see also [12]). This will allow to look for the pp and ${ }^{7} B e$ solar neutrinos by elastic neutrinoelectron scattering with a threshold of $11 \mathrm{keV}$ or at most $19 \mathrm{keV}$ (limit of possible tritium background) (Fig. 5) which would be the lowest threshold among other proposals to detect pp neutrinos, such as HERON, HELLAZ, NEON, LENS, MOON, XMASS.

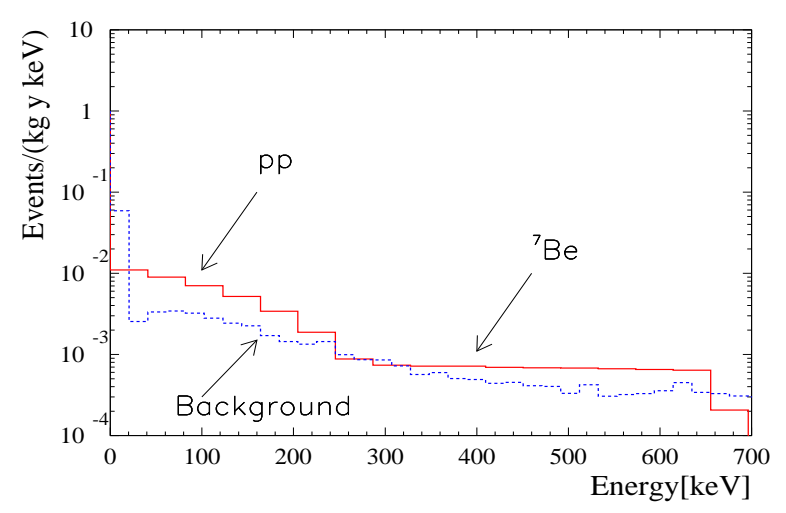

Figure 5. Simulated spectrum of low-energy solar neutrinos (according to SSM) for the GENIUS detector (1 tonne natural or enriched Ge) (from [6]).

The counting rate of GENIUS (10 ton) would be 6 events per day for pp and 18 per day for ${ }^{7} \mathrm{Be}$ neutrinos, i.e. similar to BOREXINO, but by a factor of 30 to 60 larger than a 20 ton LENS detector and a factor of 10 larger than the MOON detector.

\section{GENIUS - Test Facility}

Construction of a test facility for GENIUS GENIUS-TF - consisting of $\sim 40 \mathrm{~kg}$ of HP Ge detectors suspended in a liquid nitrogen box has been started. Up to end of 2000, three detectors each of $\sim 2.5 \mathrm{~kg}$ and with a threshold of as low as $\sim 500 \mathrm{eV}$ have been produced.

Besides test of various parameters of the GENIUS project, the test facility would allow, with the projected background of 4 events $/ \mathrm{kg}$ y $\mathrm{keV}$ in the low-energy range, to probe the DAMA evidence for dark matter by the seasonal modulation signature within about one year of measurement with $95 \%$ c.l.. Even for an initial lower mass of 20 $\mathrm{kg}$ the time scale would be not larger than three years (for details see 13.114. If using the enriched ${ }^{76} \mathrm{Ge}$ detectors of the HEIDELBERG-MOSCOW 
experiment in the GENIUS-TF setup, a background in the $0 \nu \beta \beta$ region a factor 30 smaller than in the HEIDELBERG-MOSCOW experiment could be obtained, which would allow to test the effective Majorana neutrino mass down to $0.15 \mathrm{eV}$ (90\% c.l.) in 6 years of measurement. This limit is similar to what much larger experiments aim at, at much larger time scale (see Table 1.).

\section{Conclusion}

The GENIUS project is - among the projected or discussed other third generation double beta detectors - the one which exploits this method to obtain information on the neutrino mass to the ultimate limit. Nature is extremely generous to us, that with an increase of the sensitivity by two orders of magnitude compared to the present limit, down to $\left\langle m_{\nu}>\sim 10^{-3} \mathrm{eV}\right.$, indeed essentially all neutrino scenarios allowed by present neutrino oscillation experiments can be probed.

GENIUS is the only of the new projects which simultaneously has a huge potential for cold dark matter search, and for real-time detection of lowenergy neutrinos.

\section{REFERENCES}

1. H.V. Klapdor-Kleingrothaus in Proceedings of the First International Conference on Particle Physics Beyond the Standard Model, Castle Ringberg, Germany, 8-14 June 1997, edited by H.V. Klapdor-Kleingrothaus and H.Päs, IOP Bristol (1998) 485-531 and Int. J. Mod. Phys. A 13 (1998) 3953, and J. Phys. G 24 (1998) 483 - 516.

2. H.V. Klapdor-Kleingrothaus, H. Päs and A.Yu. Smirnov, Preprint: hep-ph/0003219, (2000) and in press in Phys. Rev. D (2000).

3. H.V. Klapdor-Kleingrothaus and Y. Ramachers, Eur. Phys. J A 3 (1998) 85 - 92.

4. V.A. Bednyakov and H.V. KlapdorKleingrothaus, Phys. Rev. D62 (2000) 043524/1-9; V.A. Bednyakov and H.V. Klapdor-Kleingrothaus, Preprint: hepph/0011233 (2000).

5. H.V. Klapdor-Kleingrothaus, L. Baudis,
G. Heusser, B. Majorovits and H. Päs, GENIUS - a Supersensitive Germanium Detector System for Rare Events, Proposal, MPIReport MPI-H-V26-1999 and Preprint: hep-ph/9910205 and in Proceedings of the Second International Conference on Particle Physics Beyond the Standard Model "BEYOND'99", Castle Ringberg, Germany, 612 June 1999, edited by H.V. KlapdorKleingrothaus and I.V. Krivosheina, IOP Bristol, (2000) 915 - 1014.

6. L. Baudis and H.V. Klapdor-Kleingrothaus, Eur. Phys. J. A 5 (1999) 441-443.

7. H.V. Klapdor-Kleingrothaus, "60 Years of Double Beta Decay", World Scientific, Singapore (2001) 1253p.

8. H.V. Klapdor-Kleingrothaus, H. Päs and A.Yu. Smirnov, to be publ. (2001)

9. H.V. Klapdor-Kleingrothaus and H. Päs, Preprint: physics/0006024 and Comm. in Nucl. and Part. Phys. (2000).

10. H.V. Klapdor-Kleingrothaus, in Proc of International Symposium on Lepton and Baryon Number Violation, Trento, Italy, 20-25 April, 1998, ed H.V. Klapdor-Kleingrothaus and I.V. Krivosheina, IOP, Bristol, (1999) 251-301 and Preprint: hep-ex/9901021 and H.V. Klapdor-Kleingrothaus , Springer Tracts in Modern Physics, 163 (2000) 69 104.

11. J. Ellis, A. Ferstl and K.A. Olive, Phys. Lett. B 481 (2000) 304 - 314 and Preprint: hepph/0001005 and Preprint: hep-ph/0007113.

12. H.V. Klapdor-Kleingrothaus, in Proc. Int. Workshop on Low Energy Solar Neutrinos, LowNu2, Dec. 4-5; and in Proc. of NOON2000 Conf., Dec. 6-8 (2000) Tokyo, Japan, ed: Y. Suzuki et al. World Scientific, Singapore (2001).

13. H.V. Klapdor-Kleingrothaus, L. Baudis, A. Dietz, G. Heusser, I. Krivosheina, B. Majorovits, H. Strecker, S.T. Belyaev, V.I. Lebedev and coworkers, MPI-H-V32-2000.

14. L. Baudis, A. Dietz, G. Heusser, B. Majorovits, H. Strecker, H.V. KlapdorKleingrothaus, hep-ex/0012022, submitted for publication. 
Table 1

Some key numbers of future double beta decay experiments (and of the HEIDELBERG-MOSCOW experiment). Explanations: $\nabla$ - assuming the background of the present pilot project. $* *$ - with matrix element from [Sta90*-II], [Tom91**-I], [Hax84**-I], [Wu91*-II], [Wu92*-II] (see Table II in [HM99*-III]). $\triangle$ - this case shown to demonstrate the ultimate limit of such experiments. For details see [7].

\begin{tabular}{|c|c|c|c|c|c|c|c|}
\hline $\begin{array}{c}\beta \beta- \\
\text { Isoto- } \\
\text { pe }\end{array}$ & Name & Status & $\begin{array}{c}\text { Mass } \\
\text { (ton- } \\
\text { nes) }\end{array}$ & $\begin{array}{c}\text { Assumed } \\
\text { backgr. } \\
\dagger \text { events/ } \\
\text { kg y keV, } \\
\ddagger \text { events/kg } \\
\text { y FWHM, } \\
\text { * events } \\
\text { /yFWHM }\end{array}$ & $\begin{array}{l}\text { Run- } \\
\text { ning } \\
\text { Time } \\
\text { (tonn. } \\
\text { years) }\end{array}$ & $\begin{array}{l}\text { Results } \\
\text { limit for } \\
0 \nu \beta \beta \\
\text { half-life } \\
\text { (years) }\end{array}$ & $\begin{array}{r}<m_{\nu}> \\
(\mathrm{eV})\end{array}$ \\
\hline${ }^{76} \mathrm{Ge}$ & $\begin{array}{c}\text { HEIDEL- } \\
\text { BERG } \\
\text { MOSCOW } \\
\text { [Kla99e**] } \\
\text { [HM2000*] } \\
{[\text {-III] }} \\
\end{array}$ & $\begin{array}{l}\text { run- } \\
\text { ning }\end{array}$ & $\begin{array}{l}0.011 \\
\text { (enri- } \\
\text { ched) }\end{array}$ & $\begin{array}{l}\dagger 0.06 \\
\ddagger 0.24 \\
* 2\end{array}$ & $\begin{array}{l}35.5 \\
\mathrm{~kg} \mathrm{y}\end{array}$ & $\begin{array}{c}1.9 \cdot 10^{25} \\
90 \% \text { c.l. } \\
3.1 \cdot 10^{25} \\
68 \% \text { c.l. } \\
\text { NOW !! }\end{array}$ & $\begin{array}{c}<0.34 * * \\
\mathbf{9 0} \% \text { c.l. } \\
<0.26 * * \\
\mathbf{6 8} \% \text { c.l. } \\
\text { NOW !! }\end{array}$ \\
\hline${ }^{100} \mathrm{Mo}$ & $\begin{array}{l}\text { NEMO III } \\
\text { [NEM2000] }\end{array}$ & $\begin{array}{l}\text { under } \\
\text { constr. }\end{array}$ & $\begin{array}{r}\sim 0.01 \\
\text { (enri- } \\
\text {-ched) }\end{array}$ & $\begin{array}{c}\dagger \mathbf{0 . 0 0 0 5} \\
\ddagger 0.2 \\
* 2 \\
\end{array}$ & $\begin{array}{r}50 \\
\mathrm{~kg} y \\
\end{array}$ & $10^{24-25}$ & $0.3-0.7$ \\
\hline${ }^{130} \mathrm{Te}$ & $\begin{array}{l}\text { CUORE }^{\nabla} \\
\text { [Gui99a* } \\
\text {-VI] }\end{array}$ & $\begin{array}{l}\text { Pro- } \\
\text { posal }\end{array}$ & $\begin{array}{c}0.75 \\
\text { (natu- } \\
\text { ral) }\end{array}$ & $\begin{array}{c}\dagger 0.5 \\
\ddagger 4.5 \\
* 1000\end{array}$ & 5 & $9 \cdot 10^{24}$ & $0.2-0.5$ \\
\hline${ }^{130} \mathrm{Te}$ & CUORE & $\begin{array}{l}\text { Pro- } \\
\text { posal }\end{array}$ & $\begin{array}{c}0.75 \\
\text { (natu- } \\
\text { ral) }\end{array}$ & $\begin{array}{l}\dagger 0.005 \\
\ddagger 0.045 \\
* 45 \\
\end{array}$ & 5 & $9 \cdot 10^{25}$ & $0.07-0.2$ \\
\hline${ }^{100} \mathrm{Mo}$ & $\begin{array}{l}\text { MOON } \\
\text { [Eji99b* } \\
\text {-VI] }\end{array}$ & idea & $\begin{array}{c}10 \text { (en- } \\
\text { rich.) } \\
100 \\
\text { (nat.) } \\
\end{array}$ & $?$ & $\begin{array}{l}30 \\
300\end{array}$ & $?$ & 0.03 \\
\hline $\begin{array}{l}{ }^{136} \mathrm{Xe} \\
{ }^{136} \mathrm{Xe}\end{array}$ & $\begin{array}{c}\text { EXO } \\
\text { [Dan2000a] }\end{array}$ & $\begin{array}{l}\text { Pro- } \\
\text { posal }\end{array}$ & $\begin{array}{l}1 \\
10\end{array}$ & $\begin{array}{l}* 0.4 \\
* 0.6\end{array}$ & $\begin{array}{l}5 \\
10\end{array}$ & $\begin{array}{l}8.3 \cdot 10^{26} \\
1.3 \cdot 10^{28}\end{array}$ & $\begin{array}{l}0.05-0.14 \\
0.01-0.04\end{array}$ \\
\hline${ }^{76} \mathrm{Ge}$ & $\begin{array}{c}\text { GENIUS } \\
\text { [Kla97** } \\
-\mathrm{VI}]\end{array}$ & $\begin{array}{l}\text { Pro- } \\
\text { posal }\end{array}$ & $\begin{array}{c}1 \\
\text { (enrich.) } \\
1 \\
\end{array}$ & $\begin{array}{c}\dagger 0.04 \cdot 10^{-3} \\
\ddagger 0.15 \cdot 10^{-3} \\
\quad * 0.15 \\
\quad * 1.5\end{array}$ & $\begin{array}{r}1 \\
10 \\
\end{array}$ & $\begin{array}{l}5.8 \cdot 10^{27} \\
2 \cdot 10^{28} \\
\end{array}$ & $\begin{array}{r}0.02-0.05 \\
0.01-0.028 \\
\end{array}$ \\
\hline${ }^{76} \mathrm{Ge}$ & $\begin{array}{l}\text { GENIUS } \\
\text { [Kla97**- } \\
\text {-VI }]\end{array}$ & $\begin{array}{l}\text { Pro- } \\
\text { posal }\end{array}$ & $\begin{array}{c}10 \\
\text { (enrich.) }\end{array}$ & $\begin{array}{c}\ddagger 0.15 \cdot 10^{-3} \\
0^{\triangle}\end{array}$ & $\begin{array}{l}10 \\
10\end{array}$ & $\begin{array}{c}6 \cdot 10^{28} \\
5.7 \cdot 10^{29}\end{array}$ & $\begin{array}{c}0.006- \\
0.016 \\
0.002- \\
0.0056\end{array}$ \\
\hline
\end{tabular}




\title{
Elsevier instructions for the preparation of a 2-column format camera-ready paper in $\mathrm{AT}_{\mathrm{E} X}$
}

\author{
P. de Groot ${ }^{a *}$ and X.-Y. Wang ${ }^{\mathrm{b}}$ \\ ${ }^{a}$ Mathematics and Computer Science Division, Elsevier Science Publishers B.V., \\ P.O. Box 103, 1000 AC Amsterdam, The Netherlands \\ ${ }^{\mathrm{b}}$ Economics Department, University of Winchester, \\ 2 Finch Road, Winchester, Hampshire P3L T19, United Kingdom
}

These pages provide you with an example of the layout and style for $100 \%$ reproduction which we wish you to adopt during the preparation of your paper. This is the output from the $\mathrm{AT}_{\mathrm{E}} \mathrm{X}$ document class you requested.

\section{FORMAT}

Text should be produced within the dimensions shown on these pages: each column $7.5 \mathrm{~cm}$ wide with $1 \mathrm{~cm}$ middle margin, total width of $16 \mathrm{~cm}$ and a maximum length of $20.2 \mathrm{~cm}$ on first pages and $21 \mathrm{~cm}$ on second and following pages. The ${ }_{\mathrm{A}}^{\mathrm{A}} \mathrm{T} \mathrm{X}$ document class uses the maximal stipulated length apart from the following two exceptions (i) $\mathrm{HAT}_{\mathrm{E}} \mathrm{X}$ does not begin a new section directly at the bottom of a page, but transfers the heading to the top of the next page; (ii) $\mathrm{IT}_{\mathrm{E}} \mathrm{X}$ never (well, hardly ever) exceeds the length of the text area in order to complete a section of text or a paragraph. Here are some references: [1,2].

\subsection{Spacing}

We normally recommend the use of 1.0 (single) line spacing. However, when typing complicated mathematical text IATEX automatically increases the space between text lines in order to prevent sub- and superscript fonts overlapping one another and making your printed matter illegible.

\subsection{Fonts}

These instructions have been produced using a 10 point Computer Modern Roman. Other

\footnotetext{
*Footnotes should appear on the first page only to indicate your present address (if different from your normal address), research grant, sponsoring agency, etc. These are obtained with the \thanks command.
}

recommended fonts are 10 point Times Roman, New Century Schoolbook, Bookman Light and Palatino.

\section{PRINTOUT}

The most suitable printer is a laser printer. A dot matrix printer should only be used if it possesses an 18 or 24 pin printhead ("letter-quality").

The printout submitted should be an original; a photocopy is not acceptable. Please make use of good quality plain white A4 (or US Letter) paper size. The dimensions shown here should be strictly adhered to: do not make changes to these dimensions, which are determined by the document class. The document class leaves at least $3 \mathrm{~cm}$ at the top of the page before the head, which contains the page number.

Printers sometimes produce text which contains light and dark streaks, or has considerable lighting variation either between left-hand and right-hand margins or between text heads and bottoms. To achieve optimal reproduction quality, the contrast of text lettering must be uniform, sharp and dark over the whole page and throughout the article.

If corrections are made to the text, print completely new replacement pages. The contrast on these pages should be consistent with the rest of the paper as should text dimensions and font sizes. 


\section{TABLES AND ILLUSTRATIONS}

Tables should be made with $\mathrm{AT}_{\mathrm{E}} \mathrm{X}$; illustrations should be originals or sharp prints. They should be arranged throughout the text and preferably be included on the same page as they are first discussed. They should have a selfcontained caption and be positioned in flushleft alignment with the text margin within the column. If they do not fit into one column they may be placed across both columns (using \begin } \{ \text { table*\} or \begin\{figure*\} so that } they appear at the top of a page).

\subsection{Tables}

Tables should be presented in the form shown in Table 1. Their layout should be consistent throughout.

Horizontal lines should be placed above and below table headings, above the subheadings and at the end of the table above any notes. Vertical lines should be avoided.

If a table is too long to fit onto one page, the table number and headings should be repeated above the continuation of the table. For this you have to reset the table counter with \addtocounter $\{$ table $\}\{-1\}$. Alternatively, the table can be turned by $90^{\circ}$ ('landscape mode') and spread over two consecutive pages (first an even-numbered, then an odd-numbered one) created by means of \begin } \{ \mathrm { table } \} [ \mathrm { h } ] \text { without } a caption. To do this, you prepare the table as a separate $\mathrm{IAT}_{\mathrm{E}} \mathrm{X}$ document and attach the tables to the empty pages with a few spots of suitable glue.

\subsection{Useful table packages}

Modern $\mathrm{AT}_{\mathrm{E}} \mathrm{X}$ comes with several packages for tables that provide additional functionality. Below we mention a few. See the documentation of the individual packages for more details. The packages can be found in $\mathrm{HAT}_{\mathrm{E}} \mathrm{X}$ 's tools directory.

array Various extensions to $\mathrm{AT}_{\mathrm{E}} \mathrm{X}$ 's array and tabular environments.

longtable Automatically break tables over several pages. Put the table in the longtable environment instead of the table environment.

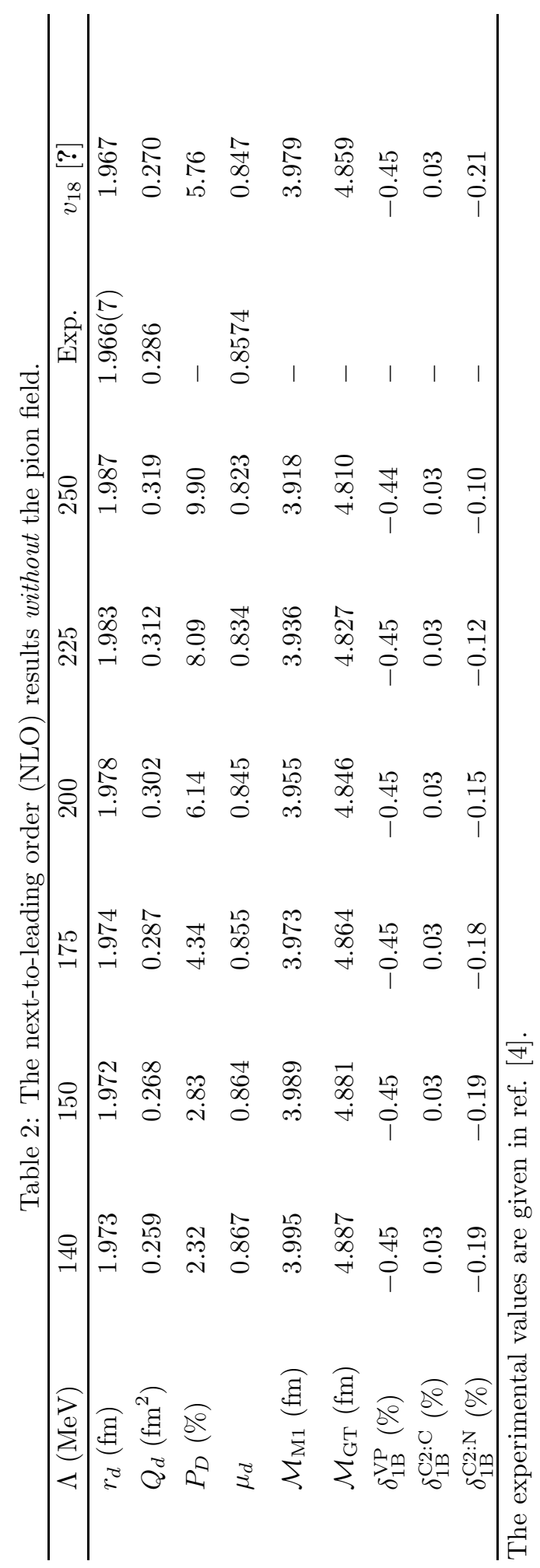


Table 1

The next-to-leading order (NLO) results without the pion field.

\begin{tabular}{lllll}
\hline$\Lambda(\mathrm{MeV})$ & 140 & 150 & 175 & 200 \\
\hline$r_{d}(\mathrm{fm})$ & 1.973 & 1.972 & 1.974 & 1.978 \\
$Q_{d}\left(\mathrm{fm}^{2}\right)$ & 0.259 & 0.268 & 0.287 & 0.302 \\
$P_{D}(\%)$ & 2.32 & 2.83 & 4.34 & 6.14 \\
$\mu_{d}$ & 0.867 & 0.864 & 0.855 & 0.845 \\
$\mathcal{M}_{\mathrm{M} 1}(\mathrm{fm})$ & 3.995 & 3.989 & 3.973 & 3.955 \\
$\mathcal{M}_{\mathrm{GT}}(\mathrm{fm})$ & 4.887 & 4.881 & 4.864 & 4.846 \\
$\delta_{1 \mathrm{~B}}^{\mathrm{VP}}(\%)$ & -0.45 & -0.45 & -0.45 & -0.45 \\
$\delta_{1 \mathrm{~B}}^{\mathrm{C} 2 \mathrm{C}}(\%)$ & 0.03 & 0.03 & 0.03 & 0.03 \\
$\delta_{1 \mathrm{~B}}^{\mathrm{C2}: \mathrm{N}}(\%)$ & -0.19 & -0.19 & -0.18 & -0.15 \\
\hline
\end{tabular}

The experimental values are given in ref. 顿.

dcolumn Define your own type of column. Among others, this is one way to obtain alignment on the decimal point.

tabularx Smart column width calculation within a specified table width.

rotating Print a page with a wide table or figure in landscape orientation using the sidewaystable or sidewaysfigure environments, and many other rotating tricks. Use the package with the figuresright option to make all tables and figures rotate in clockwise. Use the starred form of the sideways environments to obtain full-width tables or figures in a two-column article.

\subsection{Line drawings}

Line drawings should be drawn in India ink on tracing paper with the aid of a stencil or should be glossy prints of the same; computer prepared drawings are also acceptable. They should be attached to your manuscript page, correctly aligned, using suitable glue and not transparent tape. When placing a figure at the top of a page, the top of the figure should be at the same level as the bottom of the first text line.

All notations and lettering should be no less than $2 \mathrm{~mm}$ high. The use of heavy black, bold lettering should be avoided as this will look unpleasantly dark when printed.

\subsection{PostScript figures}

Instead of providing separate drawings or prints of the figures you may also use PostScript files which are included into your $\mathrm{AT}_{\mathrm{E}} \mathrm{X}$ file and printed together with the text. Use one of the packages from $\mathrm{IAT}_{\mathrm{E} X} \mathrm{X}$ 's graphics directory: graphics, graphicx or epsfig, with the \usepackage command, and then use the appropriate commands (\includegraphics or lepsfig) to include your PostScript file.

The simplest command is:

\includegraphics\{file\}, which inserts the PostScript file file at its own size. The starred version of this command:

\includegraphics*\{file\}, does the same, but clips the figure to its bounding box.

With the graphicx package one may specify a series of options as a key-value list, e.g.:

$\backslash$ includegraphics [width=15pc] \{file $\}$

$\backslash$ includegraphics [height $=5 \mathrm{pc}]\{\mathrm{file}\}$

$\backslash$ includegraphics $[\mathrm{scale}=0.6]\{\mathrm{file}\}$

$\backslash$ includegraphics [angle $=90$, width $=20 \mathrm{pc}]\{\mathrm{file}\}$

See the file grfguide, section "Including Graphics Files", of the graphics distribution for all options and a detailed description.

The epsfig package mimicks the commands familiar from the package with the same name in $\mathrm{LT}_{\mathrm{E}} \mathrm{X} 2.09$. A PostScript file file is included with the command \psfig\{file=file\}.

Grey-scale and colour photographs cannot be included in this way, since reproduction from the 
printed CRC article would give insufficient typographical quality. See the following subsections.

Figure 1. Good sharp prints should be used and not (distorted) photocopies.

Figure 2. Remember to keep details clear and large enough.

\subsection{Black and white photographs}

Photographs must always be sharp originals (not screened versions) and rich in contrast. They will undergo the same reduction as the text and should be pasted on your page in the same way as line drawings.

\subsection{Colour photographs}

Sharp originals (not transparencies or slides) should be submitted close to the size expected in publication. Charges for the processing and printing of colour will be passed on to the author(s) of the paper. As costs involved are per page, care should be taken in the selection of size and shape so that two or more illustrations may be fitted together on one page. Please contact the Technical Editor in the Camera-Ready Publications Department at Elsevier for a price quotation and layout instructions before producing your paper in its final form.

\section{EQUATIONS}

Equations should be flush-left with the text margin; $\mathrm{LAT}_{\mathrm{E}} \mathrm{X}$ ensures that the equation is preceded and followed by one line of white space. ${ }_{\mathrm{A}} \mathrm{T}_{\mathrm{E}} \mathrm{X}$ provides the package fleqn to get the flush-left effect.

$H_{\alpha \beta}(\omega)=E_{\alpha}^{(0)}(\omega) \delta_{\alpha \beta}+\left\langle\alpha\left|W_{\pi}\right| \beta\right\rangle$

You need not put in equation numbers, since this is taken care of automatically. The equation numbers are always consecutive and are printed in parentheses flush with the right-hand margin of the text and level with the last line of the equation. For multi-line equations, use the eqnarray environment. For complex mathematics, use the $\mathcal{A} \mathcal{M S}$-LATEX package.

\section{REFERENCES}

1. S. Scholes, Discuss. Faraday Soc. No. 50 (1970) 222.

2. O.V. Mazurin and E.A. Porai-Koshits (eds.), Phase Separation in Glass, North-Holland, Amsterdam, 1984.

3. Y. Dimitriev and E. Kashchieva, J. Mater. Sci. 10 (1975) 1419.

4. D.L. Eaton, Porous Glass Support Material, US Patent No. 3904422 (1975).

References should be collected at the end of your paper. Do not begin them on a new page unless this is absolutely necessary. They should be prepared according to the sequential numeric 
system making sure that all material mentioned is generally available to the reader. Use \cite to refer to the entries in the bibliography so that your accumulated list corresponds to the citations made in the text body.

Above we have listed some references according to the sequential numeric system 낸. 